\title{
Le radon dans les stations thermales : une source d'exposition aux rayonnements ionisants
}

\author{
R. AMÉON ${ }^{1}$
}

(Manuscrit reçu le 3 juillet 2002, accepté le 26 février 2003)

RÉSUMÉ La directive 96/29/Euratom a identifié le thermalisme comme étant une activité professionnelle pendant laquelle les travailleurs sont exposés à des sources naturelles de rayonnement. En effet, la radioactivité des eaux minérales est en relation directe avec la nature géologique des roches traversées par ces eaux tout au long de leur périple jusqu'à la surface. Elle se charge en divers radioéléments et notamment en radon. Les valeurs d'activité volumique de radon mesurées dans les eaux thermales françaises et étrangères s'échelonnent de la centaine à plusieurs dizaines de milliers de becquerels par litre d'eau. De par l'utilisation qui en est faite lors des soins thérapeutiques, le radon dissous dans l'eau thermale se retrouve par dégazage dans l'atmosphère intérieure des différents locaux des stations thermales. Selon le type de soins pratiqués, l'activité volumique du radon dans l'air est très variable; elle est tributaire de deux facteurs, l'alimentation en eau thermale et donc en radon et la ventilation des différents locaux. Elle est de l'ordre de plusieurs milliers de becquerels par mètre cube d'air dans les salles d'hydrothérapie et peut atteindre la centaine de milliers de becquerels par mètre cube d'air dans certaines grottes thermales. Sur ces bases, des évaluations dosimétriques, issues d'études étrangères, montrent que l'exposition des travailleurs peut atteindre plusieurs dizaines de millisieverts par an. Les rares études françaises traitant de ce sujet montrent des niveaux d'exposition au radon des travailleurs du même ordre de grandeur, démontrant ainsi la nécessité de considérer le radon comme une source importante d'exposition dans les établissements thermaux.

ABSTRACT Radon in thermal spas: a source of exposure to natural radiation.

It is a widely recognized fact that ${ }^{222} \mathrm{Rn}$ is the prime source of exposure to natural radiation as far as the population as a whole is concerned. The European Community has identified some groups of employees to be exposed to natural radiation in its 1996 Euratom directive. Such groups include people who work more particularly in thermal spas. Average Rn-levels, ranging from hundred to several tens of thousands becquerel per liter, are found in thermal groundwater. The gazeous character of ${ }^{222} \mathrm{Rn}$ and its slight solubility lead it to desorb easily from water on coming in contact with the air. The Rn concentration in the air of spas depends on a combination of 2 factors : de-emanation of radon from water and ventilation. The average $\mathrm{Rn}$ activity concentration values cover a wide range, depending on the different occupationally-defined site. The highest values are found in water therapy rooms $\left(\sim \mathrm{kBq} \mathrm{m}^{-3}\right.$ ) technical installations and thermal gallery (up to $10^{2} \mathrm{kBq} \mathrm{m}^{-3}$ ). Foreign estimations of the annual occupational dose to the staff show that radon exposure can achieve several ten millisievert. The few French studies made on this

\footnotetext{
${ }^{1}$ IRSN, DPRE/SERGD/LEIRPA, B.P. 17, 92262 Fontenay-aux-Roses Cedex, France.
} 
subject confirm those high values of effective dose received by workers. It is necessary, therefore, to consider radon as an important source of occupational exposure in thermal spas.

\section{Introduction}

Depuis ses origines, l'homme est exposé à des rayonnements ionisants, issus de diverses sources naturelles. Le radon, gaz naturel radioactif, intervient pour près du tiers de cette exposition en valeur moyenne. Cette étude, s'inscrivant dans le cadre de l'évolution des recommandations en termes de radioprotection, donne, à partir des résultats de mesures de radon dans les stations thermales françaises et étrangères disponibles dans la littérature, les niveaux d'activité volumique du radon rencontrés dans les différents postes de soins (Améon, 2001).

La crénothérapie, appelée par tradition le thermalisme, est une pratique médicale qui propose des soins basés sur l'utilisation de l'eau thermale, des vapeurs, des boues et des gaz thermaux. Selon les indications thérapeutiques, des soins de type interne (cures de boisson, irrigations vaginales, douches buccales, inhalation...) ou externe (bains, douches, couloir de marche, pédiluve, manuluve, étuve, massages...) sont pratiqués.

Comme nombre de bâtiments, les établissements thermaux sont concernés par le radon dont les origines sont le sol en contact avec les constructions et surtout l'eau thermale dans laquelle ce gaz est présent en dissolution. La présence naturelle de radon dans les eaux thermales est parfois mise à profit dans certains établissements thermaux qui pratiquent une thérapie particulière, « la thérapie radon », dans laquelle le radon est considéré comme un agent thérapeutique à part entière afin de traiter des pathologies respiratoires ou rhumatismales (Soto et Gómez, 1998). Cette thérapie est pratiquée de manière spécifique et officielle (la reconnaissance officielle d'une source thermale comme source radon impose une concentration minimale en radionucléides dans cette eau ${ }^{2}$ ) dans plusieurs stations étrangères ${ }^{3}$, comme Jachymov en République Tchèque ou Bad Steben, Bad Münster am Stein, Bad Kreuznach, Sibyllenbad et Bad Brambach en Allemagne, ou bien encore Misasa, la « Radium Spa » du Japon (Schmitz et Fritsche, 1993 ; Morinaga et al., 1984).

\footnotetext{
$23.7 \mathrm{~Bq} \mathrm{~kg}{ }^{-1}$ de radium-226 et $370 \mathrm{~Bq} \mathrm{~kg}^{~}{ }^{1}$ de radon-222 en Autriche (Steinhäusler, 1988); $185 \mathrm{~Bq} \mathrm{I}^{-1} \mathrm{de}$ radon dans l'ealu en union soviétique ; $670 \mathrm{~Bq} \mathrm{l}^{-1}$ de radon dans l'eau el $37000 \mathrm{~Bq} \mathrm{~m}^{-3}$ dans l'air en Allemagne (Schmitz et Fritsche, 1993).

${ }^{3}$ L'ancienne union soviétique esı vraisemblablement le pays où la thérapie alpha, terme officiel de la thérapie radon, a été la plus étudiée et la plus appliquée (un million de cures radon par an dans les années 70) (Tauchert, 1972; Sansoni, 1994).
} 


\section{Contexte réglementaire en France}

En France, jusqu'à la publication de l'ordonnance du 28 mars 2001, la gestion du risque lié au radon dans les bâtiments recevant du public était encadrée par deux circulaires ${ }^{4}$ basées sur les niveaux de référence (en terme d'activité volumique de radon) et sur les zones géographiques prioritaires pour la mise en place de mesure de radon. La transposition de la directive Euratom 96/29 (1996) fixant les normes de base relatives à la protection de la population et des travailleurs contre les dangers des rayonnements ionisants a permis d'introduire par voie d'ordonnance ${ }^{5}$ un nouvel article dans le code de la santé publique. Cet article (L 1333-10) qui traite des questions relatives à l'exposition aux rayonnements naturels, introduit l'obligation pour les propriétaires de lieux ouverts au public de «mettre en cuvre des mesures de surveillance de l'exposition aux rayonnements naturels, lorsque ceux-ci sont susceptibles de porter atteinte à la santé ». En application de cet article, le décret $n^{\circ} 2002-460$, concernant uniquement la protection du public ${ }^{6}$, précise les conditions de réalisation de la mesure du radon (Art. R43-10) et les modalités de diffusion des résultats obtenus (Art. R43-11). Deux arrêtés d'application sont prévus par ce décret, ils préciseront notamment les catégories d'établissement visées. La directive 96/29/Euratom ayant identifié le thermalisme comme étant une activité professionnelle pendant laquelle les travailleurs sont exposés à des sources naturelles de rayonnement tels que les produits de filiation du radon ou du thoron (cas du naturel renforcé ${ }^{7}$ ), les stations thermales devraient être inclues dans la liste des établissements concernés. Cela d'autant plus que la dernière circulaire de la Direction générale de la santé ${ }^{8}$, définissant la politique de gestion du risque lié au radon dans les établissements recevant du public et parue avant le décret $\mathrm{n}^{\circ}$ 2002-460, incite déjà les établissements thermaux à réaliser des mesures d'activité volumique de radon.

\section{Le radon dans les établissements thermaux et leur environnement}

Le radon dans les bâtiments thermaux a pour origine principale l'eau thermale mais également les gaz thermaux ainsi que le sol en contact avec la construction.

\footnotetext{
$4 \mathrm{~N}^{\circ} 99 / 289$ du 20 mai 1999 et n $99 / 46$ du 27 janvier 1999.

5 Ordonance $n^{\circ} 2001-270$ du 28 mars 2001.

6 La protection des travailleurs doit faire l'objet d'un autre decret.

7 Activités professionnelles au cours desquelles la présence de sourees naturelles de rayonnement et/ou la manipulation de substances naturellement radioactives (utilisées pour d'autres raisons que leur caractère radioactif) entraîne une augmentation notable de l'exposition des travailleurs ou des personnes du public (titre VII de la directive Euratom 96/29).

$8 \mathrm{~N}^{\circ} 2001 / 303$ du 2 juillet 2001
} 


\subsection{Le radon dans l'eau thermale}

Les eaux minérales peuvent être d'origine météorique (infiltration d'eaux de pluie dans les terrains perméables), juvénile (l'eau provient de l'écorce terrestre) ou fossile (cas des eaux liées aux gisements de sel et de pétrole) (Pomerol et Ricour, 1992). La radioactivité des eaux minérales est en relation directe avec la nature géologique des roches traversées par ces eaux tout au long de leur périple jusqu'à la surface. Les sources les plus radioactives émergent généralement des terrains anciens (Remy et Lemaitre, 1990). Cependant, les eaux profondes ne sont pas les seules, les eaux superficielles ayant pour réservoir des roches anciennes affleurantes peuvent aussi présenter une radioactivité importante. Cette radioactivité est une conséquence de la présence d'éléments radioactifs dissous des familles de l'uranium, du thorium et de l'actinium existant dans la croûte terrestre. Les teneurs en radium-226 dans lés eaux minérales varient de quelques millibecquerels à quelques becquerels par litre d'eau. L'uranium naturel est présent à hauteur du microgramme par litre d'eau. La teneur en thorium est généralement inférieure à $10 \mu \mathrm{g}^{-1}$ du fait de la faible solubilité de cet élément dans les conditions géochimiques habituelles des gisements d'eau. Comparée à certaines eaux d'Europe centrale, la majorité des eaux françaises est peu radioactive.

Un déséquilibre entre la teneur en radium-226 et celle en radon-222 est généralement constaté. Il est dû principalement à une différence de solubilité, provoquant ainsi un excès de radon pouvant être de l'ordre de $10^{4}$ (Bad Gastein, Bagnères-de-Luchon, etc.). L'enrichissement peut être également dû à la composante neptunienne ${ }^{9}$ des eaux, lorsqu'elle existe. C'est le cas de Bagnèresde-Luchon, pour laquelle une diminution de la radioactivité de ses eaux est observée en période de gel ou de sécheresse et inversement une augmentation à la fonte des neiges ou suite à des épisodes pluvieux (Caujolle et al., 1961). Ces eaux neptuniennes peuvent être à l'origine de différences d'activité volumique de radon mesurées dans des sources adjacentes (Biancotto et al., 1991).

Les valeurs d'activité volumique de radon dans les eaux thermales, mesurées à l'émergence, sont très variables et s'échelonnent sur plusieurs ordres de grandeurs allant de la centaine à plusieurs dizaines de milliers de becquerels par litre (Tab. I). Les valeurs, données dans le tableau I, sont obtenues à partir d'un échantillon ponctuel d'eau prélevé au griffon ${ }^{10}$ et analysé avec deux grandes catégories de méthodes de mesure. La première concerne l'analyse directe du radon dans l'eau par spectrométrie gamma via les descendants émetteurs gamma

\footnotetext{
${ }^{9}$ Composante de l'eau ayant une origine superficielle en opposition à la partie plutonienne d'origine profonde.

${ }^{10}$ Point d'émergence d'une source.
} 


\section{TABLEAU I}

Activité volumique de radon, $A_{\text {vé }}$, mesurée dans les eaux thermales à l'émergence. Radon concentration, $A_{\mathrm{ve}}$, in thermal water measured at the source.

\begin{tabular}{|c|c|c|c|c|}
\hline$\cdots$ Pays & 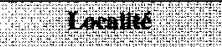 & Source. & Nxt 40 & 414 rope \\
\hline \multirow[t]{5}{*}{ France } & Bugnères-de-Luchon & Lepape 1 & 1369 & Grandpierre et al. (1962) \\
\hline & & & 1370 & Rémy et lemaitre (1990) \\
\hline & Evaux-les-bains & César & 269 & Améon el al. (2000) \\
\hline & St-Amand-les-Eaux & Vaubian & 1406 & Fontan et al. (1980) \\
\hline & Bad Münster & Maximilianquelle & 260 & Sansoni (1994) \\
\hline \multirow[t]{2}{*}{ Allernagne } & Bad Steben & Tempelquelle & 2190 & \multirow{2}{*}{ Sansoni (1997) } \\
\hline & Bad Brambach & Obere Grenzquelle & 3737 & \\
\hline Autriche & Bud Hofgastein & Elisabethquelle & 1850 & Deetjen (1997) \\
\hline Italie & Abano & $\begin{array}{c}\text { Ermitage } \\
\text { Tullio }\end{array}$ & $\begin{array}{l}481 \\
145\end{array}$ & Biancotto et al. (1991) \\
\hline URSS / Oural & Uwildy & & 66600 & Tauchert (1972) \\
\hline République tchèque & Jachymov & Step spring & 68000 & Spurnŷ (1980) \\
\hline
\end{tabular}

(Rémy et Lemaitre, 1990 ; Biancotto et al., 1991). La seconde catégorie combine un transfert du radon, dissous dans l'eau dans une phase air par dégazage, puis l'analyse du radon dégazé par des techniques classiques de mesure en atmosphère (fiole scintillante, chambre d'ionisation, etc.) (Améon et al., 2000 ; Grandpierre et al., 1962 ; Sansoni, 1997). Toutes ces méthodes présentent des sensibilités différentes mais dans la gamme de concentration rencontrée dans les eaux thermales, elles donnent des résultats équivalents (cas de Bagnères-de-Luchon), si l'on exclut les incertitudes associées au prélèvement, phase la plus délicate de la mesure du fait du caractère très mobile de ce gaz. Des teneurs très élevées sont généralement rencontrées dans les pays où les eaux sont issues d'anciennes mines d'uranium (Spurnŷ, 1980). La solubilité du radon est une fonction inverse de la température de l'eau, les eaux chaudes contiennent donc moins de radon que les eaux froides provenant de formations géologiques comparables.

Le transfert de l'eau thermale des griffons aux différents postes d'utilisation dans une station peut provoquer par dégazage une diminution de la teneur en radon initiale. Le stockage plus ou moins prolongé de l'eau thermale dans des réservoirs favorise nettement ce phénomène. Une perte d'activité en radon d'environ $80 \%$ peut être mesurée entre l'émergence et les bains (Fontan et al., 1980 ; Améon et al., 2000). En revanche, lors de l'alimentation directe des postes de soins (buvettes, étuve, etc.) par l'eau issue des griffons, la teneur en radon dans l'eau reste stable. 


\subsection{Le radon dans les gaz thermaux}

Au griffon, les gaz thermaux se dégagent spontanément des sources minérales en proportion parfois très importante par rapport à l'eau. Dans les provinces carbogazeuses, il peut se dégager jusqu'à quatre fois plus de gaz que de liquide à l'émergence et ceci avec des débits assez considérables $\left(150 \mathrm{~m}^{3} \mathrm{~h}^{-1}\right.$ à Royat). Associés à ces gaz thermaux, on peut trouver du radon ainsi que du thoron à des teneurs de l'ordre de la dizaine de milliers de becquerels par mètre cube (Degranges et collaborateurs, 1978).

\subsection{Le radon dans l'atmosphère intérieure des stations thermales}

L'activité volumique de radon dans l'atmosphère intérieure des stations thermales est tributaire de deux facteurs : la source de radon (avec généralement deux composantes : l'alimentation en eau thermale et le sol en contact avec le bâtiment) et la ventilation des différents locaux. Les valeurs les plus faibles sont mesurées en période estivale et dans les établissements récents dotés de système de ventilation mécanique. Un établissement thermal se compose généralement de trois grandes parties : les galeries techniques, les bureaux administratifs et les locaux où sont appliqués les soins. Cette dernière catégorie comprend notamment les salles d'hydrothérapie (bains, douches, massage sous-eau, piscine et couloir de marche, etc.), les salles de soins par inhalation, les galeries thermales (vaporarium, émanatorium, etc.).

Le tableau II donne quelques exemples d'activité volumique mesurée ${ }^{11}$ dans différentes salles d'établissements thermaux français et étrangers. Les atmosphères les plus riches en radon se trouvent dans les galeries techniques, qui situées souvent au sous-sol des bâtiments thermaux sont des endroits mal ventilés, et dans les salles liées à l'hydrothérapie du fait du dégazage important du radon dissous dans l'eau pendant les soins. Ce dégazage est accéléré par l'insufflation d'air comprimé dans les baignoires (cas des aérobains). L'activité volumique de radon mesurée dans ces salles varient au cours de la journée en fonction de l'utilisation de l'eau thermale (renouvellement continuel de l'eau des bains) et de la ventilation des locaux (Fig. 1). Le hall des buvettes, abritant une salle de repos ou un hall d'accueil, possède des fontaines desquelles s'écoule en permanence de l'eau, issue des griffons, destinée à la boisson. L'apport de radon, dû à l'écoulement de l'eau, y est continuel et contribue à enrichir l'atmosphère en radon jusqu'à atteindre des valeurs d'activité volumique non négligeables. Les galeries et les grottes thermales sont des sites naturels qui possèdent des

\footnotetext{
${ }^{\prime \prime}$ En l'absence d'information sur le type de mesure de radon (intćgré ou ponctuel) réalisé par les différents auteurs, aucune distinction n'est faite dins ce tableau.
} 


\section{TABLEAU II}

Activité volumique du radon, $A_{v}$, dans l'air mesurée dans différentes salles de stations thermales. Radon activity concentration, $A_{v}$, in air measured in different rooms of treatment facilities.

\begin{tabular}{|c|c|c|c|c|}
\hline Wratum & $4 \mathrm{Gror}^{2}$ & 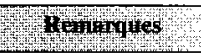 & 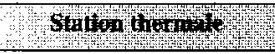 & 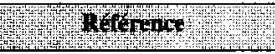 \\
\hline Bâtiment & $<40-410$ & & Espagne & Soto et Gómez $(1999)$ \\
\hline administratif & $30-220$ & & Bad Gastein (Autriche) & Lettner et al. (1996) \\
\hline Galeries techniques & $>100000$ & & & Schtnitz et Frische (1993) \\
\hline Hall des buvettes & 280 & Salle de repos & Bad Münster (Allemagne) & Sansoni (1998) \\
\hline & 1491 & & Misasa (Japon) & Morinaga et al. (1984) \\
\hline Halls des bains & $4300-7000$ & Jout - nuit & Rudas (Hongrie) & Szerbin (1996) \\
\hline & 22000 & & $\begin{array}{c}\text { Bagnères-de-Luchon } \\
\text { (France) }\end{array}$ & Giandpierte ef al. (1962) \\
\hline Aérobains & $\begin{array}{c}1300 \\
150\end{array}$ & $\begin{array}{l}\text { Sans ventilation } \\
\text { Avec ventilation }\end{array}$ & Evaux-les-Bains (France) & $\begin{array}{l}\text { Améon et al. (2000) } \\
\text { Améon et al. }(2001)\end{array}$ \\
\hline & $40-5200$ & Différentes stations & Espagne & Soto and Gómes (1999) \\
\hline & $40-5000$ & Hors - avec soins & Radenci (Yougoslavie) & Kobal et Renier (1987) \\
\hline Galeries thermates & 166000 & & Bad Gastein (Autriche) & Uzunov et al. (1981) \\
\hline & 2700 & & Misasa (Japon) & Morinaga ef al. (1984) \\
\hline Grottes thermales & 20000 & Vajeur estivale & Hospital (Hongric) & Secrbin (1996) \\
\hline & 14000 & Valeur estivale & Bagnères-de-Luchon & Grandpierre er al. (1962) \\
\hline Etuves & 7500 & & Evaux-les-Bains (France) & Améon et al. (2000) \\
\hline & 103000 & Masques & Las Caldas (Espagne) & Soto et al. (1995) \\
\hline Salles d'inhalation & 128000 & $\begin{array}{c}\text { d'inhalation } \\
\text { (thérapie radon) }\end{array}$ & Bad Mïnster (Allemagne) & Sansoni (1998) \\
\hline
\end{tabular}

atmosphères chaudes, humides et fortement chargées en radon utilisées dans le traitement de pathologies rhumatismales. Certaines grottes non isolées de l'extérieur montrent des variations saisonnières de la teneur en radon avec des valeurs fortes en été et plus faibles en hiver du fait de contraste thermique entre l'intérieur et l'extérieur de la grotte. Dans les stations thermales, l'étuve, pièce non ventilée, permet de recomposer une ambiance typique de ces galeries thermales où l'activité volumique du radon est fonction de la teneur en radon de l'eau minérale issue des griffons. Dans les établissements pratiquant «la thérapie radon », il existe des salles d'inhalation dans lesquelles le curiste respire, par l'intermédiaire d'un masque, un mélange d'air, de radon à forte concentration et parfois d'aérosols de tailles diverses (Soto et al., 1995 ; Sansoni, 1998).

Les hôtels thermaux, généralement situés à proximité des établissements de soins, sont souvent alimentés par l'eau thermale de la station et l'utilisation des baignoires dans les chambres, contribue donc à enrichir l'ambiance en gaz radon. Un raisonnement par analogie entre ces hôtels et l'habitat classique permet 


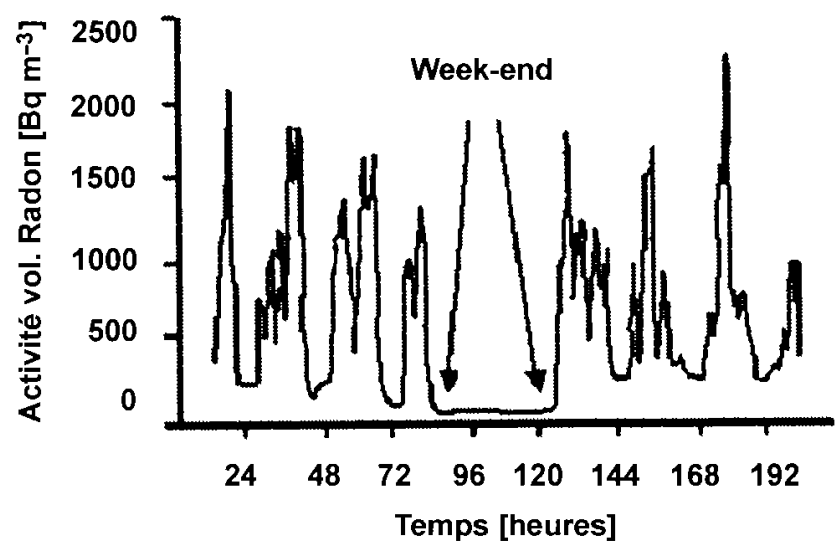

Figure 1 - Variation temporelle de l'activité volumique du radon dans une salle de soins d'une station thermale sur une période d'une semaine (d'après Lettner et aL, 1996).

Temporal variation for radon activity concentration at a treatment facility of a thermal spa over a week (adapted from Lettner et al., 1996).

d'estimer que $10 \mathrm{~Bq}^{-1}$ de radon-222 dans 1'eau contribuent à générer $1 \mathrm{~Bq} \mathrm{m^{-3 }}$ de radon dans l'air intérieur (Nazaroff et al., 1987 ; UNSCEAR, 1993).

\subsection{Descendants à vie courte du radon dans l'atmosphère intérieure des établissements thermaux}

Comme les autres gaz rares, le radon inhalé, du fait de sa très faible réactivité chimique, a peu d'affinités avec le milieu biologique et est presque entièrement réexhalé. En revanche, ses descendants à vie courte, constitués d'atomes radioactifs, sous forme d'aérosols solides, ont un fort potentiel énergétique. Une fois inhalés, ils se déposent le long des voies respiratoires et délivrent leur énergie alpha aux cellules et tissus proches du site de dépôt. Bien que la quantification de ces produits de filiation du radon soit une phase importante dans le processus de l'évaluation des doses reçues, peu de travaux sur la mesure des descendants dans les établissements thermaux sont disponibles dans la littérature car la mise en œuvre des techniques de mesure classiques des descendants du radon (prélèvement d'aérosols sur filtre) est difficile dans les conditions de forte humidité relative qui règnent dans ces atmosphères.

Tout comme pour le radon gaz, les variations journalières de l'activité volumique des descendants dans l'atmosphère d'une station thermale sont 

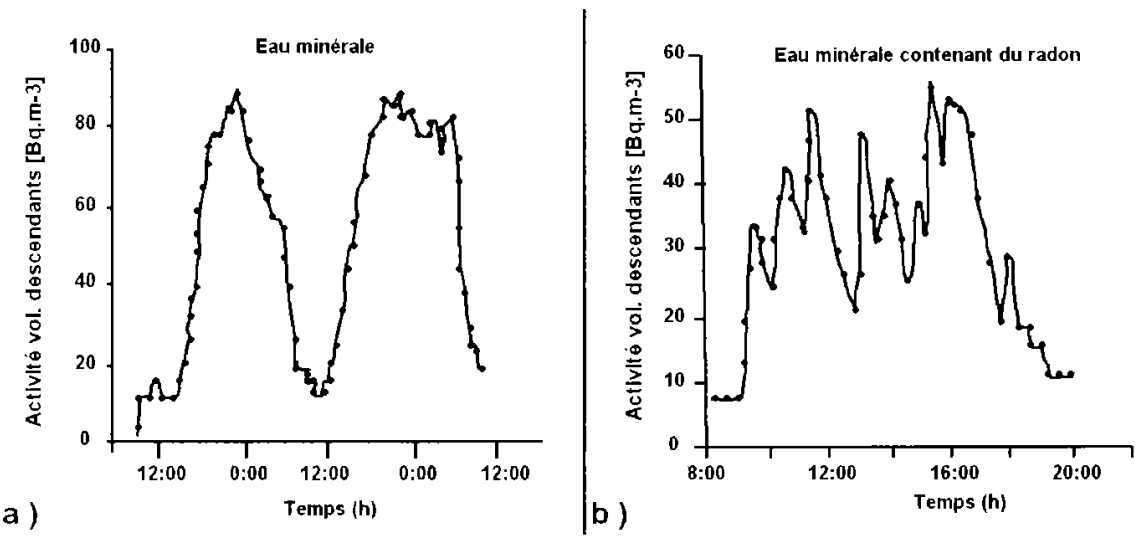

Figure 2 - Mesure en continu de la concentration des descendants à vie courte du radon dans deux salles d'hydrothérapie sur 48 heures (a) et sur 12 heures (b) (d'après Schmitz et Fritsche, 1993).

Continuous measurement of airborne radon progeny at two working places in balneotherapy over 48 hours (a) and over 12 hours (b) (adapted from Schmitz et Fritsche, 1993).

importantes et sont dépendantes de l'activité thermale et de la ventilation des locaux. La figure 2 montre les variations temporelles de l'activité volumique des descendants mesurées dans deux salles d'hydrothérapie dont seule la seconde (Fig. 2b) est alimentée avec une eau riche en radon. Dans le premier cas, le radon est principalement issu du sol en contact avec le bâtiment et l'augmentation de l'activité volumique des descendants est due à l'arrêt de la ventilation et au confinement des locaux après les soins matinaux (Fig. 2a). Dans le second cas, l'apport en radon et en descendants est dû à l'utilisation de l'eau thermale pendant les bains et le signal mesuré permet de bien distinguer la durée des soins égale à 30 minutes (Fig. 2b).

Le facteur d'équilibre, $F$, qui traduit le déséquilibre radioactif entre le radon et ses descendants à vie courte varie fortement selon le type d'activité thermale pratiquée dans les salles concernées (Tab. III). Il semble être plus élevé dans les galeries thermales que dans les salles d'hydrothérapie dans lesquelles la ventilation mécanique élimine les descendants. Une évolution saisonnière du facteur d'équilibre corrélée avec la variation saisonnière de l'activité volumique du radon dans les grottes a été mise en évidence avec des valeurs plus élevées l'été que l'hiver (Vaupotič et al., 2001).

$F$ tend à diminuer lorsque le taux d'humidité relative augmente (Reichelt, 1996). Ainsi, dans certaines salles d'hydrothérapie, le remplissage des bains engendre une augmentation de l'activité volumique du radon tandis que celle en 
TABLEAU III

Facteur d'équilibre mesuré dans différentes salles d'établissements thermaux. Equilibrium factor measured in different rooms of thermal spas.

\begin{tabular}{|c|c|c|}
\hline \multicolumn{3}{|c|}{ 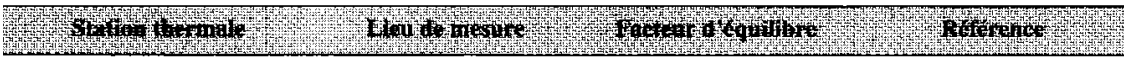 } \\
\hline $\begin{array}{l}\text { Bad Gastcin } \\
\text { (Autriche) }\end{array}$ & $\begin{array}{l}\text { Galeric Thermalc } \\
\text { Salles hydrothérapie }\end{array}$ & Lettner el al. (1996) \\
\hline $\begin{array}{c}\text { Bad Mutnster } \\
\text { (Aflemagne) }\end{array}$ & $\begin{array}{l}\text { Cloche inhalation } \\
\text { Sálles hydrothérape }\end{array}$ & Sansoni $(19) 8)$ \\
\hline Hongrie & $\begin{array}{l}\text { Grottes naturelles } \\
\text { et tunnel arificiel }\end{array}$ & Szerbin (1996) \\
\hline $\begin{array}{l}\text { Postojna } \\
\text { (Slovente) }\end{array}$ & $\begin{array}{l}\text { Grotte naluelle } \quad 0,42 \leq F \leq 0,69 \text { en hiver } \\
0,33 \leq F \leq 0,86 \text { en }\end{array}$ & Vapotic at al. $(2001)$ \\
\hline Bagnères-de-Luchon (France) & $\begin{array}{l}\text { Galerie « Bordeu» } \\
\text { Poste de humage }\end{array}$ & Grandpierre et al. (1962) \\
\hline
\end{tabular}

descendants reste faible en raison de la forte humidité générée et de l'augmentation de la ventilation mécanique hygroréglable. $F$ atteint alors son maximum lorsque la teneur en radon est la plus faible, l'activité volumique des descendants montrant une évolution inverse de celle du radon (Lettner et al., 1996).

La faible valeur du facteur d'équilibre mesurée dans les cloches à inhalation de Bad Münster est due à la filtration des descendants réalisée en amont du système. Cette filtration n'est pas toujours effective (Italie et Autriche) mais en raison des dépôts d'aérosols dans les canalisations des postes d'inhalation ou de humage, $F$ est généralement inférieur à 0,5 . Soto et Gómez ont montré un facteur d'équilibre variable selon le descendant pris en compte $\left(0,8\right.$ pour le ${ }^{218}$ Po et entre 0,2 et 0,6 pour les autres) (Soto et Gómez, 1999).

\subsection{Le radon dans l'environnement extérieur des établissements thermaux}

La stratégie de gestion du risque lié au radon porte l'intérêt sur les bâtiments, où le radon se trouve généralement en concentration plus importante que dans l'atmosphère extérieure en raison des plus faibles taux de renouvellement d'air qui y règnent. Plus spécifiquement, les données concernant les teneurs en radon dans l'atmosphère extérieure des établissements thermaux sont peu nombreuses et il est parfois difficile de savoir si les valeurs annoncées sont représentatives du site ou si elles ne sont le reflet que de points singuliers. Toutefois, la présence d'un grand nombre de sources thermales dans une région engendre généralement une augmentation de la radioactivité atmosphérique. Ces régions (comme les complexes thermaux de Bad Gastein (Autriche) et de Misasa (Japon)) sont alors 
identifiées comme « zones à haut niveau de radioactivité naturelle » (High Levels of Natural Radiation Areas). Ainsi dans la région de Misasa, forte de ses 70 sources thermales ${ }^{12}$, il est estimé que $130 \times 10^{7} \mathrm{~Bq}$ de radon diffusent chaque jour de l'eau et du sol. L'activité volumique moyenne de radon dans l'atmosphère extérieure de cette station thermale est deux fois supérieure à celle mesurée dans l'environnement d'un site dépourvu de sources thermales chaudes (Morinaga et al., 1984). Une activité volumique de radon de l'ordre de $30 \mathrm{~Bq} \mathrm{~m}^{-3}$ équivalente à celle de Misasa, a été mesurée dans le parc thermal et le jardin de l'hôtel de Luchon (Grandpierre et al., 1962) tandis que la concentration moyenne de radon dans l'environnement extérieur est estimée à $10 \mathrm{~Bq} \mathrm{~m}^{-3}$ à l'échelle mondiale (UNSCEAR, 2000). Les promenades dans les parcs thermaux étant généralement une des activités intégrantes des cures, il peut donc paraître opportun d'étudier plus avant l'environnement extérieur des établissements thermaux pour une meilleure prise en compte de l'exposition des curistes - et en règle générale du public - au radon et à ses produits de filiation.

\section{Aspect dosimétrique}

L'inhalation des descendants à vie courte du radon constitue la principale source d'exposition loin devant l'ingestion de l'eau thermale radioactive. Les évaluations de doses dues au radon sont généralement effectuées sur la base des résultats des mesures intégrées du radon sur plusieurs mois. Mais en raison des fortes variations temporelles des teneurs en radon et en descendants dans l'atmosphère des stations, ces mesures intégrées peuvent sous-estimer de 20 à $50 \%$ la teneur en radon dans l'air pendant la seule période d'application des soins lorsque la principale source de radon dans la station est l'eau thermale (Améon et al., 2000). Elles peuvent en revanche la surestimer dans le cas où le radon, issu essentiellement du sol en contact avec le bâtiment, s'accumule dans les locaux lors du confinement nocturne et de l'arrêt de la ventilation. L'utilisation de dosimètres individuels permet donc une estimation plus précise de la dose reçue à chaque poste de travail. Le tableau IV montre quelques valeurs de dose efficace calculées pour différentes catégories de personnel des établissements thermaux étrangers (médecins, masseurs, agents thermaux, techniciens de surface, etc.) ainsi que pour les curistes. Les calculs réalisés par les différents auteurs tiennent compte du temps de présence dans l'établissement thermal, qui correspond au nombre d'heures de travail pour le personnel (la majorité des stations a une activité saisonnière et généralement estivale). Ces estimations montrent que l'exposition annuelle des travailleurs thermaux peut être conséquente et atteindre quelques dizaines de millisieverts.

\footnotetext{
${ }^{12}$ Ces sources délivrent chaque jour 3 millions de litre d’eau dont l'acrivité volumique moyenne de radon est de 4.37 Bप I ${ }^{\prime}$
} 


\section{TABLEAU IV}

Dose efficace calculée pour le personnel et les curistes de différentes stations thermales. Effective annual dose estimated for employees and patients in different thermal spas.

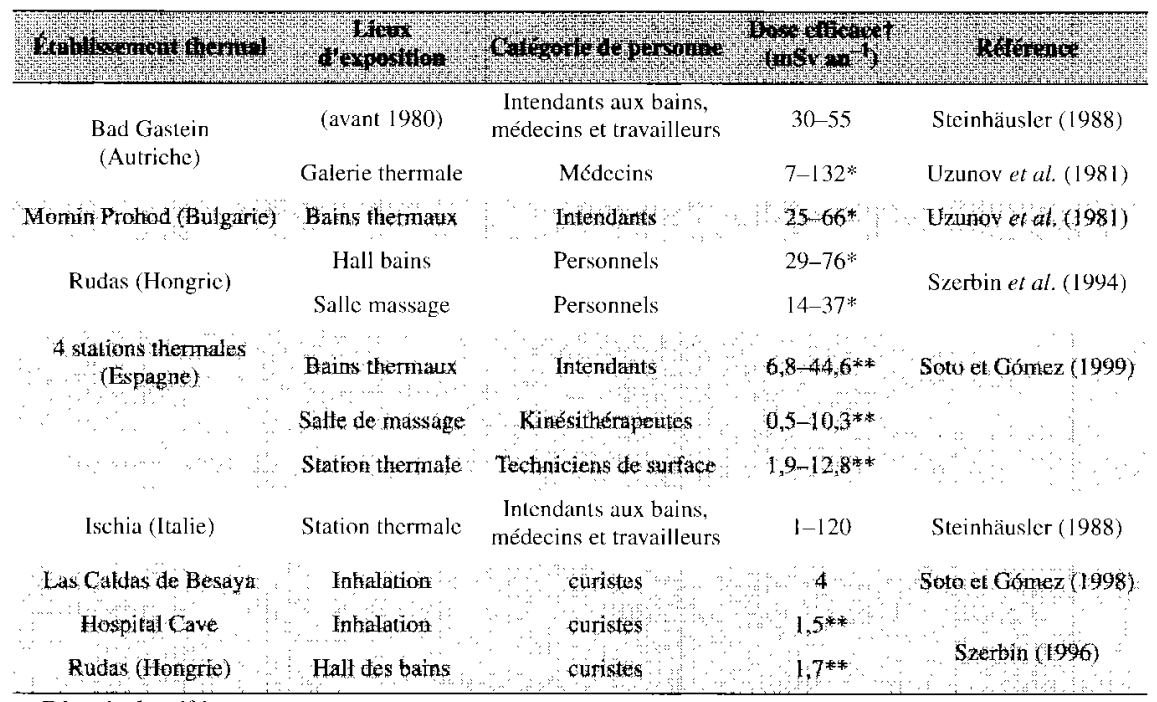

† D'après la référence, * calculé d'après ICRP 32, ** calculé d'après ICRP 65.

Il est à noter que toutes les estimations de dose efficace sont obtenues à partir de modèles mathématiques (ICRP, 1981, 1993) qui utilisent différents facteurs de pondération (tissulaire pour le poumon, du rayonnement alpha et des effets de débit de dose) dont certains semblent être mal estimés. Ainsi, le facteur de pondération des particules alpha égal à 20 semble être surévalué d'un facteur 2 à 3 (Birchall et James, 1994 ; Monchaux et Masse, 1999 ; Marsh et al., 2002). Dans l'hypothèse d'une réduction de ce facteur, les estimations de doses correspondantes seraient évidemment réduites d'autant.

Les doses élevées ne sont pas l'apanage des stations thermales qui présentent des eaux riches en radon, la ventilation est également un facteur important (Soto et Gómez, 1999). Ainsi, les techniciens de surface qui interviennent souvent lorsque la ventilation est arrêtée peuvent être exposés significativement (Améon, 2001).

L'évaluation des doses reçues par les patients lors d'un séjour en station ${ }^{13}$ n'est pas simple à appréhender en raison des difficultés à reconstituer le déroulement

\footnotetext{
${ }^{13}$ Généralement, les cures sont de l'ordre de trois semaines (duréc nécessaire à l'efficacité thérapeutique des cures en France).
} 
des soins lors d'une cure (durée des traitements, temps de présence à chaque poste thérapeutique, présence ou non de descendants à vie courte du radon lors des traitements (par inhalation notamment)). En revanche, il est possible d'apprécier la dose efficace associée à un type de soins pendant la cure (Tab. IV).

\section{Conclusion}

Cette étude bibliographique montre que l'activité volumique de radon mesurée dans les eaux thermales peut atteindre plusieurs milliers de becquerels par litre. L'utilisation de l'eau thermale en crénothérapie engendre par dégazage de très fortes concentrations de radon dans l'atmosphère intérieure des salles de soins des établissements thermaux. Dans des conditions de ventilation défavorables voire inexistantes, il n'est pas rare de mesurer des teneurs en radon de l'ordre de plusieurs milliers de becquerels par mètre cube d'air. Ces fortes valeurs d'activité volumique de radon, accompagné ou non de ses descendants à vie courte, peuvent conduire, selon des études étrangères, à une exposition du personnel de l'ordre de la dizaine à quelques dizaines de millisieverts par an. L'exposition des curistes au radon est généralement inférieure à $2 \mathrm{mSv} a^{-1}$ mais peut parfois s'avérer non négligeable du point de vue de la radioprotection. Les rares études françaises traitant de ce sujet montrent des niveaux d'exposition des travailleurs au radon du même ordre de grandeur que ceux obtenus à l'étranger, démontrant ainsi la nécessité d'étudier plus avant la situation des établissements thermaux au regard du radon.

\section{RÉFÉRENCES}

Améon R. (2001) Radon et thermalisme. Étude bibliographique, Rapport DPRE/SERGD/01-34, IPSN

Améon R., Bcncïto A., Thoreux A. (2000) Étude de l'activité volumique en radon dans une station thermale française, Cas d'Evaux-les-bains. Rapport DPRE/SERGD/00-51, IPSN.

Améon R., Beneïlo A., Thoreux A. (2001) Mesures de l'activité volumique de radon dans le nouvel établissement thermal d'Evaux-les-bains, Rapport DPRE/SERGD/01-58, IPSN.

Biancotı R., Lafisca S., Lucchese R., Martinelli C., Predicatori F., Rosa M., Tacconi A., Trotti F. (1991) Radon concentration in mineral and thermal waters of veneto: an estimate of ingestion and inhalation doses. Rad. Prot. Dosim. 36, 129-135.

Birchall A., James A.C. (1994) Uncertainty analysis of the effective dose per unit cxposure from radon progeny and implications for ICRP risk-weighting factors, Rad. Prot. Dosim. 53(1-4), 133-140.

Caujolle F., Auvergnat R., Oustrin J. (1961) Régime radioactil de l'aire d'ćmergence luchonnaise. Société Française d'Hydrologie et de Climatologie Médicales, 95-99.

Dectjen P. (1997) Scientific Principles of the Health Treatments in Bad Gastein and Bad Holgastein. Sem. Reports Salzburg - Austria ISSV 0256-4173.

Degranges P. et collaborateurs (1978) Étude chimique et isotopique de l'eau minérale el des gaz thermaux de Royat (P.-de-D.), Rapport BRGM, code SGN064 MCE. 
Directive 96/29 (1996) Euratom du conseil du 13 mai 1996, fixant les normes de bases relatives à la protection sanitaire de la population et des travailleurs contre les dangers résultants des rayonnements ionisants.

Fontan M., Caridroit M., Erb F., Tauziède-castel C. (1980) Quelques études sur l'action des caux, boues et gaz thermaux radioactifs de Saint-Amand-les-eaux, J. Fr. Hydrol. 11, 55-62.

Grandpierre R., Pellerin P., Arnaud Y., Blanquet P., Moroni J. (1962) La radioactivité des eaux et gaz de Bagnères-de-Luchon, Ann. Inst. Hydrol. Climat. 33, 22-39.

ICPR Publication 32 (1981) Limits for Inhalation of Radon Daughters by Workers. Ann. ICRP 6(1).

ICPR Publication 65 ( 1993 ) Protection against radon-222 at home and at work. Ann. ICRP 23(2).

Kobal I., Renier A. (1987) Radioactivity of the atomic spa at Podčetrtek, slovenia, Yugoslavia, Health Phys. 53, 307-310.

Lettner H., Hubmer A.K., Rolle R., Steinhäusler F. (1996) Occupational exposure to radon in treatment facilities of the radon-spa Badgastein, Austria, Environm. Intem. 22, suppl. 1, S399-S407.

Marsh J.W. et al. (2002) Uncertainty analysis of the weighted equivalent lung dose per unit exposure to radon progeny in the home, Rad. Prot. Dosim. 102(3), 229-248.

Monchaux G., Masse R. (1999) Les incertitudes sur la dose délivrée aux voies respiratoires après exposition au radon, Radioprotection 34(3), 367-387.

Morinaga H., Mifume M., Furuno K. (1984) Radioactivity of water and air in Misasa spa, Japan, Rad. Prot. Dosim. 7(1-4), 295-297.

Nazaroff W.W., Doyle S.M., Nero A.V., Sextro R.G. (1987) Potable water as a source of airborne 222Rn in US dwellings. A review and assessment, Health Phys. 52, 281-295.

Pomerol C., Ricour J. (1992) Terroirs et Thermalisme de France, Éditions du BRGM, Orléans.

Reichelt A. (1996) Radon and its decay products in water purification plants, Environm. Intern. 22, suppl. 1, S761-S768.

Remy M.L., Lemaitre N. (1990) Eaux minérales et radioactivité, Hydrogéologie 4, 267-278.

Sansoni B. (1994) Selected Topics in Radon Balneology, Europe 1993/94: Springs, Spas, Therapy, Radiation Protection, 47. Jahrestagung der Japanischen Gesellschaft für Balneologie, pp. 30-31.

Sansoni B. (1997) Planungen Radonheilbad Naturpark Fichtelgebirge, Internat. Environ. Consult. Newsletter 3, 40-41.

Sansoni B. (1998) Radon-222 und Radonfolgeprodukte in der Luft der Inhalations- und Bädertherapie in Bad Münster am Stein-Ebernburg, Internat. Environ. Consult. Newsletter 4, 5667.

Schmitz J., Fritsche R. (1993) Radon exposure at Workplaces. Final Report of a survey in German Balneotherapy. Austrian-Italian-Hungarian Radiation Protection Symposium. Radiation in neighbouring countries in Central Europe, pp. 345-348.

Soto J., Fernández P.L., Quindós L.S., Gómez-Arozamena J. (1995) Radioactivity in Spanish spas, Sci. Tot. Environm. 162, 187-192.

Soto J., Gómez J. (1998) Radon Balneology in Spain, Internat. Environ. Consult. Newsletter, 4, 48-55.

Soto J., Gómez J. (1999) Occupational Doses from Radon in Spanish Spas, Health Phys. 76, 398-401.

Spurnŷ Z. (1980) A New Approach to the Dosage in Radon Water Spas, Jaderná Energie 26, 140-142.

Steinhäusler F. (1988) Radon spas: Source term, doses and risk assessment, Rad. Prot. Dosim. 24, $257-259$.

Szerbin P. (1996) Natural radioactivity of certain spas and caves in Hungary, Environm. Intern. 22, suppl. 1, S389-S398. 


\section{LE RADON DANS LES STATIONS THERMALES}

Szerbin P., Köteles G., Stúr D. (1994) Radon concentrations in Rudas thermal bath, Budapest, Rad. Prot. Dosim. 56(1-4), 319-321.

Tauchert D. (1972) Aspekte der Radon-Therapie in der sowjetunion Bericht über einen Studienaufenthalt in der UdSSR, Z. Physioth. 24, 73-79.

UNSCEAR (1993) United Nations. Sources, Elfects and Risks of Ionizing Radiation. Report to the General Assembly, with annexes, New York.

UNSCEAR (2000) United Nations. Sources, Ellects and Risks of Ionizing Radiation. Report to the General Assembly, with annexes, New York.

Uzunov 1., Steinhäusler F., Pohl E. (1981) Carcinogenic risk of exposure to radon daughters associated with radon spas, Health Phys. 41(6), 807-813.

Vaupotič J., Csige I., Radolič V., Hunyadi I., Planinič J., Kobal I. (2001) Methodology of radon monitoring and dose estimates in Postojna cave, Slovenia, Health Phys. 80(2), 142-147. 